UDC 517.3

\author{
E. A. Shcherbakov, I. A. Avdeyev
}

\title{
ON THE COMPACTNESS OF ONE CLASS OF QUASICONFORMAL MAPPINGS
}

\begin{abstract}
We consider an elliptic system in the disk $|z|<1$ for the so-called $p$-analytic functions. This system admits degeneration at the boundary of the disk. We prove compactness of the family of $K$-quasiconformal mappings, which are the solutions of the uniformly elliptic systems approximating the degenerating one.

Key words: quasi-conformal mappings, sobolev spaces, elliptic systems, embedding theorems, topological mappings, Dirichlet integral, Douglas integral, harmonic functions
\end{abstract}

2010 Mathematical Subject Classification: 30C70, 30C75

Introduction. Let us consider in the disk $D=|z|<1$ an elliptic system of the following form:

$$
\left\{\begin{array}{l}
p u_{x}=v_{y} \\
p u_{y}=-v_{x} .
\end{array}\right.
$$

It is well known [2] (see also [5]), that for the function $p$, strictly positive in the closure of the disk, there exist normalized topological mappings that satisfy system (1). In spite of the simplicity of system (1), the problem of existence of topological mappings generated by the solutions of the degenerate system (1) is rather difficult. M. Lavrentiev [4] proposed to approximate the degenerate system (1) by uniformly elliptic systems and thus to find quasiconformal mappings with unbounded characteristics corresponding to the degenerate system (1). It is at this stage that the question of compactness of the $K_{n}$-quasiconformal mappings corresponding to unbounded family $\left\{K_{n}\right\}$ does arise.

The main result. Let $p=p(z), z \in\{|z|<1\}$ be a measurable function, such that

$$
p(z)>0, \quad z \in D, \quad \inf _{z \in D} p(z)=0
$$

(C) Petrozavodsk State University, 2019 
We suppose also that the following condition for the function $p$ is fulfilled:

$$
p(z)=p(-\bar{z}), z \in D \text {. }
$$

Let $p_{n}(z):=p\left(\left(1-\frac{1}{n}\right) z\right)$ and $w_{n}=u_{n}+i v_{n}$ be solutions of the systems

$$
\left\{\begin{array}{l}
p_{n} \alpha_{x}=\beta_{y} \\
p_{n} \alpha_{y}=-\beta_{x} .
\end{array}\right.
$$

They perform normalized topological mappings of the disk $D$ onto itself. As it has been already said, solutions of this kind exist. We may assume that they are normalized by the correspondence

$$
w_{n}(-i)=-i, \quad w_{n}(1)=1, \quad w_{n}(i)=i .
$$

It is clear that we can consider the mappings $w_{n}$ as the extensions of the mappings of the set

$$
\bar{D} \cap\{\operatorname{Re} z \geqslant 0\}
$$

onto itself to the whole disk by the symmetry. Our goal is to prove the following theorem:

Theorem 1. Let $\left\{w_{n}\right\}, w_{n}=u_{n}+i v_{n}$ be the constructed above sequence of topological maps of the disk $D$ onto itself, normalized by conditions

$$
w_{n}(1)=1, \quad w_{n}(-1)=-1, \quad w_{n}(i)=i .
$$

Then $\left\{w_{n}\right\}$ is compact in the sense of uniform convergence in $|z| \leqslant 1$.

Proof. It is known [6] that a sequence of topological mappings of bounded domains is compact in the sense of uniform convergence if their Dirichlet integrals are uniformly bounded. It is easy to prove that the Dirichlet integrals of the functions $v_{n}$ are uniformly bounded. Indeed, taking into account (4), we arrive at the following estimate:

$$
\begin{aligned}
& \iint_{D}\left[\left(v_{n x}\right)^{2}+\left(v_{n y}\right)^{2}\right] d x d y=\iint_{D}\left[-p_{n} u_{n y} v_{n x}+p_{n} u_{n x} v_{n y}\right] d x d y= \\
& =\iint_{D} p_{n}\left[u_{n x} v_{n y}-u_{n y} v_{n x}\right] d x d y=\iint_{D} p_{n} J_{w n} d x d y \leqslant \max [p] * \operatorname{mes}[D] .
\end{aligned}
$$


Here $J_{w n}$ denotes the Jacobian of the mapping $w_{n}$. Now, the $\inf _{z \in D} p(z)$ being equal to zero, we cannot pursue the same way in order to evaluate Dirichlet integrals for the functions $u_{n}$. Still, we can show that the Dirichlet integrals for the functions $u_{n}$ are also uniformly bounded. According to the Slobodetsky embedding theorem [3], we have the inequality that relates the norm of the functions $\phi$ in the Sobolev space $W^{1,2}(D)$ and the integral $\mathcal{D}(\phi)$ of Douglas:

$$
\mathcal{D}(\phi)=\iint_{D} \frac{\left|\phi\left(z_{1}\right)-\phi\left(z_{2}\right)\right|^{2}}{\left|z_{2}-z_{1}\right|^{2}} d z_{1} d z_{2} .
$$

This inequality is

$$
\|\phi\|_{W^{1,2}(D)} \leqslant c_{0} \mathcal{D}(\phi) .
$$

Here $c_{0}$ is some universal constant. The functions $u_{n}^{2}$ evidently belong to the space $W^{1,2}(D)$. Applying now inequality (7) to these functions, we get

$$
\begin{aligned}
& \left\|u_{n}^{2}\right\|_{W^{1,2}(D)} \leqslant c_{0} \mathcal{D}\left(u_{n}^{2}\right)=c_{0} \iint_{\delta D \delta D} \frac{\left|u_{n}^{2}\left(z_{1}\right)-u_{n}^{2}\left(z_{2}\right)\right|^{2}}{\left.\left|z_{2}-z_{1}\right|^{2}\right]} d z_{1} d z_{2}= \\
& =c_{0} \iint_{\delta D \delta D} \frac{\left|v_{n}^{2}\left(z_{1}\right)-v_{n}^{2}\left(z_{2}\right)\right|^{2}}{\left|z_{2}-z_{1}\right|^{2}} d z_{1} d z_{2} \leqslant 4 c_{0} \iint_{\delta D \delta D} \frac{\left|v_{n}^{2}\left(z_{1}\right)-v_{n}^{2}\left(z_{2}\right)\right|^{2}}{\left|z_{2}-z_{1}\right|^{2}} d z_{1} d z_{2} .
\end{aligned}
$$

According to the result of Douglas [1], the Dirichlet integral and the Douglas integral coincide for the harmonic functions. Let $h_{n}$ be a harmonic function from the space $W^{1,2}(D)$ coinciding with $v_{n}$ on the boundary of the disk. Then the Dirichlet integral $D\left(h_{n}\right)$ for the function $h_{n}$ is less than that for the function $v_{n}$. This means that

$$
\begin{aligned}
& \left\|u_{n}^{2}\right\|_{W^{1,2}(D)} \leqslant 4 c_{0} \iint_{D} \frac{\left|v_{n}\left(z_{1}\right)-v_{n}\left(z_{2}\right)\right|^{2}}{\left|z_{2}-z_{1}\right|^{2}} d z_{1} d z_{2}= \\
& \quad=4 c_{0} \mathcal{D}\left(h_{n}\right) \leqslant 4 c_{0} \mathcal{D}\left(v_{n}\right) \leqslant 4 c_{0} \max [p] * \operatorname{mes}[D] .
\end{aligned}
$$

Now let us consider topological mappings $W_{n}$ :

$$
W_{n}:=u_{n}^{2}+i v_{n}
$$


Using inequalities (7) and (9), we establish that the functions $W_{n}$ have uniformly bounded Dirichlet integrals. Now, we prove that the sequence $\left\{W_{n}\right\}$ is a sequence of topological mappings of $D$ to a bounded domain. The functions $v_{n}=W_{n}$ on the imaginary line represent injective mapping of the line. Thus, it remains only to show that the functions $W_{n}$ realize injective mapping in the set $\{z \in D \mid \operatorname{Re} z>0\}$. Assume the contrary, i. e., that there exists $z_{1}, z_{2} \in\{z \in D \mid \operatorname{Re} z>0\}$, such that

$$
\left(u_{n}^{2}+i v_{n}\right)\left(z_{1}\right)=\left(u_{n}^{2}+i v_{n}\right)\left(z_{2}\right) .
$$

Now we have two possibilities:

$$
u_{n}\left(z_{1}\right)=u_{n}\left(z_{2}\right) \wedge\left(v_{n}\left(z_{1}\right)=v_{n}\right)\left(z_{2}\right)
$$

and

$$
u_{n}\left(z_{1}\right)=-u_{n}\left(z_{2}\right) \wedge\left(v_{n}\left(z_{1}\right)=v_{n}\right)\left(z_{2}\right) .
$$

The points $u_{n}\left(z_{1}\right)+i v_{n}\left(z_{1}\right)$ and $u_{n}\left(z_{2}\right)+i v_{n}\left(z_{2}\right)=-u_{n}\left(z_{1}\right)+i v_{n}\left(z_{1}\right)$ cannot coincide when $\{z \in D \mid \operatorname{Re} z>0\}$. Thus, the second condition is impossible. The first one leads to the conclusion that $z_{1}=z_{2}$. All this means that the uniformly bounded functions $W_{n}$ realize topological mappings, their Dirichlet integrals being uniformly bounded. It means that $\left\{W_{n}\right\}$ is compact [5] in the sense of uniform convergence in a closed circle. It is clear that convergence $\left\{W_{n}\right\}$ implies convergence $\left\{w_{n}\right\}$ in the unit disk. Indeed, assume that it is not so. Then there exists a sequence $\left\{z_{n}\right\}$, such that $\lim _{n \rightarrow \infty}\left(z_{n}\right)=z_{*}, \lim _{n \rightarrow \infty}\left(w_{n}\left(z_{n}\right)\right) \neq w\left(z_{*}\right)$. The sequence $\left\{v_{n}\right\}$ converges uniformly in $\bar{D}$ and inside $\bar{D} \backslash\{u=0\}$. That means that $\lim _{n \rightarrow \infty}\left(\operatorname{Re} z_{n}\right)=\operatorname{Re} z_{*}=0$ and $\lim _{n \rightarrow \infty}\left(u_{n}^{2}\left(z_{n}\right)=0\right.$. The functions $u=u(z), u^{2}=u^{2}(z)$ has null boundary values on the imaginary axis. It means that

$$
\lim _{n \rightarrow \infty}\left(w_{n}\left(z_{n}\right)\right)=\lim _{n \rightarrow \infty}\left(v_{n}\left(z_{n}\right)\right)=v\left(z_{*}\right)=u\left(z_{*}\right)+i v\left(z_{*}\right)=w\left(z_{*}\right) .
$$

The contradiction we arrive at signifies uniform convergence of $w_{n}$ in $\bar{D}$. The theorem is proved.

Remark. The compactness of the sequence of topological mappings $\left\{w_{n}\right\}$ has been proved for the any degeneration of an elliptic system (1) on the boundary. 


\section{References}

[1] Douglas J. Solution of the problem of Plateau., Trans. Amer. Math. Soc., 1967, vol. 33(1931), pp. $61-112$.

DOI: https://doi.org/10.1090/S0002-9947-1931-1501590-9

[2] Gergen J. J., Dressel F. G. Mapping by p-regular functions. Duke math. J., 1951, vol. 18 , no. 1 , pp. 185-210. DOI: 10.1215/S0012-7094-51-01815-7

[3] Kufner A., Oldrich J., Fuchik S. Function spaces. Academia, P., 1977.

[4] Lavrentiev M. A., Shabat B. V., Problems of hydrodynamics and their mathematical models, Izdatel'stvo Nauka, Moscow, 1973 (in Russian).

[5] Sauvigny F. Partial Differential Equations 2. Functional Analytic Methods. XVI, Springer, 2012.

[6] Suvorov G. D. Families of flat topological mappings, Edit. of. Siber. Dep. USSR Acad. Sci., Novosibirsk, 1965 (in Russian).

Received July 09, 2019.

In revised form, October 29, 2019.

Accepted October 30, 2019.

Published online November 4, 2019.

Kuban State University

149 Stavropolskaya str., Krasnodar 350040, Russia

E-mail: avdeyev.iv@gmail.com 